Editorial

\title{
New Definition of Fetal Growth Restriction: Consensus Regarding a Major Obstetric Complication
}

\section{Nova definição de restrição do crescimento fetal: Consensos para uma importante complicação obstétrica}

\author{
Luciano Marcondes Machado Nardozza ${ }^{1}$ Ana Cristina Perez Zamarian ${ }^{1}$ Edward Araujo Júnior ${ }^{1}$ \\ ${ }^{1}$ Department of Obstetrics, Escola Paulista de Medicina, Universidade \\ Federal de São Paulo, São Paulo, SP, Brazil
}

Rev Bras Ginecol Obstet 2017;39:315-316.

Fetal growth restriction (FGR) affects 5-10\% of all pregnancies, and it is the second leading cause of perinatal mortality, accounting for $\sim 30 \%$ of stillbirths; it leads to premature births and intrapartum asphyxia. ${ }^{1}$ Fetuses with FGR have an increased risk for perinatal morbidity and mortality, impaired neurological and cognitive development during childhood and adolescence, and cardiovascular and endocrine disorders in adulthood. $^{2}$

Fetal growth restriction is characterized by cases wherein the fetus does not achieve full intrauterine growth and development because of impaired placental function. ${ }^{1}$ However, in clinical practice, FGR is difficult to define, and there is currently no gold standard for its diagnosis. One of the greatest challenges is the differentiation between small for gestational age (SGA) fetuses, who are constitutionally small and healthy, and restricted fetuses, who present with some degree of placental dysfunction and an increased risk for adverse perinatal outcomes. ${ }^{3}$

In both the literature and clinical practice, many authors and medical schools use different concepts to define FGR on the basis of biometric factors (weight percentile), Doppler (umbilical artery, middle cerebral artery, uterine artery, and ductus venosus), and biochemical markers (primarily placental growth factor - PIGF). In 2016, a multicenter team of international FGR experts conducted a study based on the Delphi method in an attempt to establish a consensus regarding the definition of early and late FGR. ${ }^{4}$ The study included questionnaires with four phases, and the results were reported to the participants after each phase. In the first phase, the distinction between early and late FGR was defined. The second and third stages discussed the parameters that could be separately considered for diagnosing FGR and those that had to be considered along with other parameters to conclude a diagnosis. In the final stage, some possible algorithms were presented to experts, and the algorithm with the highest number of votes was considered the final algorithm for defining FGR. ${ }^{2}$ The consensus concluded that the cutoff value between early and late FGR would be gestational age (GA) of 32 weeks, and the following parameters were used to define FGR in the absence of fetal malformations: early FGR ( $<32$ weeks): (i) fetal abdominal circumference below the third percentile for GA OR estimated fetal weight below the third percentile for GA OR zero diastole of the umbilical artery on Doppler (isolated criteria) and (ii) estimated fetal weight or waist circumference below the tenth percentile for GA AND the pulsatility index of the uterine and umbilical arteries above the 95th percentile for GA (combined parameters) and late FGR ( $\geq 32$ weeks): (i) fetal abdominal circumference below the third percentile for GA OR estimated fetal weight below the third percentile for GA and (ii) the combination of at least two of the following parameters: (a) estimated fetal weight or fetal abdominal circumference below the tenth percentile for GA, (b) the reduction of more than two quartiles in the growth curve, and (c) the cerebroplacental association below the fifth percentile for GA or the pulsatility index of the umbilical artery above the 95th percentile for GA.

Establishing an accurate diagnosis of FGR is fundamental both in the obstetric clinic to improve the detection of fetuses at an increased risk for adverse perinatal outcomes, and in scientific investigations to standardize concepts and enable further discoveries. Although the consensus based on the Delphi method establishes definitions that appear appropriate and consistent with recent studies, we should remember that it is a consensus based only on expert opinion, which requires scientific evidence to be ratified.
Address for correspondence Edward Araujo Júnior, PhD, Department of Obstetrics, Escola Paulista de Medicina, Universidade Federal de São Paulo, Rua Belchior de Azevedo, 156, apto. 111, 05089-030, Torre Vitória, São Paulo, SP, Brazil (e-mail: araujojred@terra.com.br).
DOI https://doi.org/ 10.1055/s-0037-1603741. ISSN 0100-7203.
Copyright $\odot 2017$ by Thieme Revinter Publicações Ltda, Rio de Janeiro, Brazil
License terms

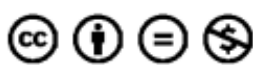


The choice of 32 weeks as the cutoff value between early and late FGR appears appropriate because hypertrophy of fetal cells initiates approximately at this GA. Moreover, this GA is the most commonly used age in the main study on FGR. ${ }^{4}$ Some criticism regarding the parameters defined in the consensus included the use of uterine artery Doppler as a parameter associated with birth weight below the tenth percentile for diagnosing early FGR because uterine artery Doppler exhibited low sensitivity in a meta-analysis on the prediction of adverse perinatal events in FGR. ${ }^{5}$ The cerebroplacental association in FGR has been extensively investigated. ${ }^{6,7}$ The combined Doppler of the middle cerebral and umbilical arteries improves detection sensitivity relative to that of isolated Doppler. Furthermore, studies have demonstrated a relation between changes in the cerebroplacental association and increased morbidity and mortality in late FGR. ${ }^{8}$ However, although the consensus described above establishes that the ratios below the fifth percentile are considered to be altered, a cutoff value has not been defined.

We also believe that biochemical methods may be used for diagnosing FGR in the near future, thereby increasing sensitivity. ${ }^{9}$ The current consensus does not rely on any biochemical assays for determining the diagnosis. Furthermore, the importance of certain sonographic parameters, including the volume of amniotic fluid and fetal weight curve that needs to be used for each population, needs to be better established for FGR to ensure improved management and perinatal prognosis.

The definition of FGR remains controversial in the medical literature. Therefore, it is essential to establish and standardize diagnostic concepts, and although expert consensus based on the Delphi method appears promising, it still remains to be validated for use in the clinical practice. An accurate identification of restricted fetuses enables proper monitoring and better decision making at the time of delivery and reduces the risk for fetal deaths because clinical treatment of FGR remains unavailable.

\section{References}

1 Nardozza LM, Caetano AC, Zamarian AC, et al. Fetal growth restriction: current knowledge. Arch Gynecol Obstet 2017; 295(05):1061-1077

2 Nardozza LM, Araujo Júnior E, Barbosa MM, Caetano AC, Lee DJ, Moron AF. Fetal growth restriction: current knowledge to the general Obs/Gyn. Arch Gynecol Obstet 2012;286(01):1-13

3 Hanley GE, Janssen PA. Ethnicity-specific growth distributions for prediction of newborn morbidity. J Obstet Gynaecol Can 2012; 34(09):826-829

4 Gordijn SJ, Beune IM, Thilaganathan B, et al. Consensus definition of fetal growth restriction: a Delphi procedure. Ultrasound Obstet Gynecol 2016;48(03):333-339

5 Velauthar L, Plana MN, Kalidindi M, et al. First-trimester uterine artery Doppler and adverse pregnancy outcome: a meta-analysis involving 55,974 women. Ultrasound Obstet Gynecol 2014; 43(05):500-507

6 DeVore GR. The importance of the cerebroplacental ratio in the evaluation of fetal well-being in SGA and AGA fetuses. Am J Obstet Gynecol 2015;213(01):5-15

7 Arabin B, Goerges J, Bilardo CM. The importance of the cerebroplacental ratio in the evaluation of fetal well-being in SGA and AGA fetuses. Am J Obstet Gynecol 2016;214(02):298-299

8 Figueras F, Gratacós E. Update on the diagnosis and classification of fetal growth restriction and proposal of a stage-based management protocol. Fetal Diagn Ther 2014;36(02):86-98

9 Karagiannis G, Akolekar R, Sarquis R, Wright D, Nicolaides KH. Prediction of small-for-gestation neonates from biophysical and biochemical markers at 11-13 weeks. Fetal Diagn Ther 2011; 29(02):148-154 